\title{
FIBERED STABLE COMPACTA HAVE FINITE HOMOTOPY TYPE
}

\author{
ROSS GEOGHEGAN ${ }^{1}$
}

\begin{abstract}
It is proved that a fibered compact metric space having the shape of a CW complex has the homotopy type of that complex, and that its Wall obstruction to finiteness is zero.
\end{abstract}

1. Introduction and statement of theorems. The following problem is wellknown and of long standing (compare [20, Appendix] or [12]):

PROBLEM. Let the compact topological space $X$ be homotopy equivalent to a (CW) complex. Must $X$ be homotopy equivalent to a finite complex?

The answer is known to be "yes" if $X$ is a compact metric ANR [19], and "yes" if and only if the Wall obstruction of $X$ vanishes [18]. It is unknown whether the obstruction can fail to vanish when $X$ is compact.

Let us call a compactum ( $\equiv$ compact metric space) fibered if it is homeomorphic to an inverse limit proj $\lim _{n}\left\{\ldots X_{n} \stackrel{f_{n}}{\leftarrow} X_{n+1} \ldots\right\}$ in which each $X_{n}$ is a compactum homotopy equivalent to a finite complex, and each $f_{n}$ is a (Hurewicz) fibration.

TheOREM 1.1. A fibered compactum $X$ which is homotopy equivalent to a complex is homotopy equivalent to a finite complex.

The proof is in \$2. It consists of using the Serre spectral sequence to show that, for each large $n, X$ is homotopy equivalent to a certain compact covering space of $X_{n}$. (Serre fibrations would do, in Theorem 1.1, but not in the rest of the paper.)

Theorem 1.1 is obviously related to the Problem, but it acquires more interest when combined with Theorem 1.2, for which we need the following definition. A compactum $X$ is shape equivalent to a (possibly infinite) complex $L$ if there is a homotopy commutative diagram of maps

Received by the editors October 26, 1977 and, in revised form, December 14, 1977.

AMS (MOS) subject classifications (1970). Primary 55F40, 54E60; Secondary 55D99, 55D10, $55 \mathrm{H} 10$.

Key words and phrases. Homotopy type of a CW complex, shape, Wall obstruction, sequence of fibrations.

'Supported in part by National Science Foundation Grants MCS 75-10377 and MCS 77-00104.

${ }^{2}$ Added in proof. S. Ferry has-announced a negative solution to the Problem.

(๑) American Mathematical Society 1978 


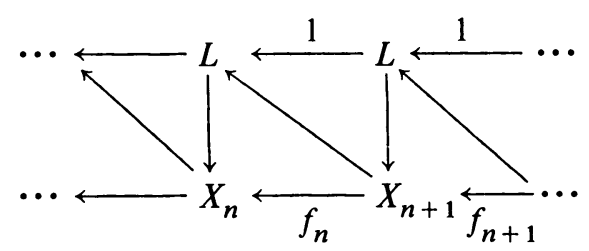

where each $X_{n}$ is a compactum homotopy equivalent to a finite complex, and $X$ is homeomorphic to $\operatorname{proj} \lim _{n}\left\{X_{n}\right\}$. Starting from this definition, no knowledge of shape theory is required for this paper, but we should state some facts (for references see Remark 2.1, below): (i) this definition of "shape equivalent to a complex" is equivalent to the usual ones; (ii) up to cofinal subsequences, this definition does not depend on the choice of sequence whose inverse limit is $X$; (iii) if $X$ is homotopy equivalent to $L$, then $X$ is shape equivalent to $L$. (ii) implies that if $X$ is also fibered, we may assume w.l.o.g. that the maps $f_{n}$ in $(\dagger)$ are fibrations.

THEOREM 1.2. A fibered compactum, which is shape equivalent to a complex, is homotopy equivalent to that complex.

This is an easy consequence of $[6,3.4 .1]$. The deduction is in $\$ 3$.

Compacta shape equivalent to complexes are often said to be stable. The title of this paper, therefore, summarizes Theorems 1.1 and 1.2. Note that fibered compacta can fail to be stable (e.g., the dyadic solenoid), and stable compacta can fail to be homotopy equivalent to complexes (e.g., the Warsaw circle). It is the combination of "fibered" and "stable" which is nice. Criteria for recognizing stable compacta can be found in [4], [5] and [8]. Their geometrical properties are studied in [15].

Returning to the Problem, we recall that there are compacta shape equivalent to complexes but not shape equivalent to finite complexes [4]. They are defined as inverse limits of finite complexes: let $X=\operatorname{proj} \lim _{n}\left\{X_{n} ; f_{n}\right\}$ be one such, shape equivalent to a complex $L$. One would like to change this sequence into a sequence of compact fibrations, while maintaining shape equivalence to $L$. If one could, then Theorem 1.2 would give a negative solution to the Problem. But Theorem 1.1 shows that this cannot be done.

Therefore, if there is a compactum $X$ homotopy equivalent to a complex, but not to a finite complex, it cannot be built as the limit of an inverse sequence of spaces of finite homotopy type and fibrations. ${ }^{3}$ (Dually, using the fact that surjective cofibrations are homeomorphisms [17], it is easy to show that $X$ cannot be built as the limit of a direct sequence of spaces of finite homotopy type and cofibrations.)

We conclude with a consequence of the proofs of 1.1 and 1.2. It gives some geometrical insight, since one does not expect the inverse limit of ANR's to be an ANR; the proof is in $\S 4$ :

THEOREM 1.3. Let $X=\operatorname{proj}^{\lim _{n}}\left\{X_{n} ; f_{n}\right\}$ where $X_{n}$ is a compact metric

\footnotetext{
${ }^{3}$ See footnote 2.
} 
$A N R$ and $f_{n}$ is a fibration, for all $n$. If $X$ is shape equivalent to a complex, then $X$ is a compact metric ANR.

These results were announced in [7].

2. Proof of Theorem 1.1. We are given $X=\operatorname{proj} \lim _{n}\left\{X_{n} ; f_{n}\right\}$ where each $f_{n}$ is a fibration and $X_{n}$ is compact and homotopy equivalent to a finite complex. We may assume each $X_{n}$ path-connected. If we were willing to assume each $X_{n}$ locally path-connected we could proceed to use covering space theory on the $X_{n}$ 's (since $X_{n}$ is certainly semilocally 1-connected). We avoid this by embedding each $X_{n}$ as a weak deformation retract of a compact ANR $Y_{n}$ (e.g., if $X_{n}$ is homotopy equivalent to the finite simplicial complex $K$, let $Y_{n}$ be $K \times\left(\right.$ Hilbert Cube)). Covering spaces of $Y_{n}$ will contain the covering spaces of $X_{n}$ which we need. By the homotopy extension property, there is an inverse system $\left\{Y_{n} ; g_{n}\right\}$ where $g_{n}$ agrees with $f_{n}$ on $X_{n+1}$. We will also need $X$ to be embedded as a weak deformation retract of a noncompact ANR, $Y$. An elementary way to do this is: $X \times($ circle) is homotopy equivalent to a finite simplicial complex $K$ [11]. So, by [16, p. 76] there is a weak homotopy equivalence $h: \bar{K} \rightarrow X \times R$, where $\bar{K}$ is an infinite cyclic covering space of $K$. Since $X$ has the (unpointed!) homotopy type of a complex, $h$ is a homotopy equivalence (care is needed if the base point of $X$ is bad). Now let $Y$ be $\bar{K} \times$ (Hilbert Cube), and approximate the inverse of $h$ by an embedding. $X \times\{0\}$ is then correctly embedded in $Y$.

REMARK 2.1 (not used in the sequel). Suppose each $X_{n}$ is embedded as a $Z$-set in the Hilbert Cube. Then, by [2, Theorem 25.2], $X_{n}$ is a weak deformation retract of arbitrarily small compact ANR neighborhoods. Hence we can pick $Y_{n}$ in such a way that proj $\lim _{n}\left\{Y_{n} ; g_{n}\right\}=\operatorname{proj} \lim _{n}\left\{X_{n} ; f_{n}\right\}=$ $X$. It then follows from [10] and [13] that our definition of shape equivalence is standard; see (i)-(iii) in $\$ 1$. Alternatively, we could use [15].

We will need three lemmas, presumably all folklore.

LEMMA 2.2. Let $F \hookrightarrow E \rightarrow B$ be a fibration. If $E$ and $B$ are homotopy equivalent to complexes, so is $F$. If $E$ is compact, $E, B$ and $F$ are all dominated by finite complexes.

SketCH OF PROOF. Consider the fibration $G \hookrightarrow F^{\prime} \rightarrow E$ where $F^{\prime}$ and $G$ are homotopy equivalent to $F$ and the loop space of $B$, respectively. By [12], $G$ is homotopy equivalent to a complex, hence, by [3], $F^{\prime}$ is too, hence also $F$. The last sentence of the lemma is now obvious.

If $T$ is a space, let $\operatorname{dim}_{Z} T \in N \cup\{\infty\}$ be the least element such that the singular homology $H_{i}(T)$ with $Z$-coefficients is zero for all $i>\operatorname{dim}_{Z} T$.

LEMMA 2.3. Let $F \hookrightarrow E \rightarrow B$ be an orientable fibration where $B$ is pathconnected. Let $s \equiv \operatorname{dim}_{Z} B<\infty$ and $t \equiv \operatorname{dim}_{Z} F<\infty$. Then $\operatorname{dim}_{Z} E \geqslant s+$ $t$.

SKETCH OF PROOF. A straightforward computation in the Serre spectral 
sequence [16, Chapter 9]. The $E_{s+1, t}^{2}$ term may be nonzero, but $0 \neq E_{s, t}^{\infty}$ is a subgroup of $H_{s+t}(E)$.

LEMMA 2.4. Let $\bar{Y} \rightarrow Y$ be a fibration with unique path lifting, where $\bar{Y}$ is connected and $Y$ is homotopy equivalent to a finite-dimensional complex $K$. Then $\operatorname{dim}_{Z} \bar{Y}<\infty$.

Proof. For an appropriate connected covering space $\bar{K}$ of $K$, there is a weak homotopy equivalence $\bar{K} \rightarrow \bar{Y}$, by $[16$, p. 76]; and $\bar{K}$ is finitedimensional.

Proof of Theorem 1.1. Notation was set up before Remark 2.1. Choose a base point $* \in X \subset Y$ and let $p_{n}(*)$ be the base point of $X_{n} \subset Y_{n}$, where $p_{n}$ : $X \rightarrow X_{n}$ is the natural map. $p_{n}$ is a fibration; let its fiber be $F_{n} . X$ is semilocally contractible (i.e., small open sets contract in $X$ ), and $\bigcap_{n} F_{n}=$ $\left\{*\right.$. Since $X$ is compact, $F_{n}$ has small diameter when $n$ is large, so w.l.o.g. we will assume every $F_{n}$ contracts in $X$. Hence $X$ is path-connected. By Lemma 2.2, $\pi_{0}\left(F_{n}\right)$ is finite; exactness gives: $p_{n \#}: \pi_{1}(X) \rightarrow \pi_{1}\left(X_{n}\right)$ is a monomorphism and im $p_{n \#}$ has finite index in $\pi_{1}\left(X_{n}\right)$. There is a map $q_{n}: Y \rightarrow Y_{n}$ extending $p_{n}$, and a compact connected covering space $\bar{Y}_{n} \rightarrow Y_{n}$ corresponding to the subgroup im $q_{n \#}$. Letting $\bar{X}_{n} \rightarrow X_{n}$ be the restricted covering projection, we get a lift $\bar{p}_{n}: X \rightarrow \bar{X}_{n}$ of $p_{n}$, such that $\bar{p}_{n \#}: \pi_{1}(X) \rightarrow \pi_{1}\left(\bar{X}_{n}\right)$ is an isomorphism, $\bar{X}_{n}$ is compact and connected, and $\bar{X}_{n}$ is homotopy equivalent to a finite complex. We will show that $\bar{p}_{n}$ is a weak homotopy equivalence when $n$ is large.

Let $\tilde{X}=\operatorname{proj} \lim _{n}\left\{\tilde{X}_{n} ; \tilde{f}_{n}\right\}$. Here $\tilde{X}_{n} \rightarrow X_{n}$ is the covering projection obtained by restricting the universal cover $\tilde{Y}_{n} \rightarrow Y_{n}$, all base points are induced by $* \in X$, and $\tilde{f}_{n}$ is the restriction of $\tilde{g}_{n}$. Each $\tilde{f}_{n}$ is a fibration, hence the natural map $\tilde{p}_{n}: \tilde{X} \rightarrow \tilde{X}_{n}$ is a fibration. There is an induced fibration with unique path lifting $\tilde{X} \rightarrow X$. $\tilde{p}_{n}$ covers $\bar{p}_{n}$. There is a fibration $\bar{f}_{n}: \bar{X}_{n+1} \rightarrow \bar{X}_{n}$ covering $f_{n}$ and covered by $\tilde{f}_{n}$. Let $\bar{F}_{n}$ and $\bar{G}_{n}$ be the fibers of $\bar{p}_{n}$ and $\bar{f}_{n}$, and let $\tilde{F}_{n}$ and $\tilde{G}_{n}$ be the fibers of $\tilde{p}_{n}$ and $\tilde{f}_{n}$.

We claim $\operatorname{dim}_{Z} \tilde{F}_{n}<\infty$. To see this note: $\bar{F}_{n}$ is path-connected (exactness), $\tilde{X}$ is path-connected (since each $\tilde{X}_{n}$ is 1-connected), hence $\tilde{F}_{n}$ is path-connected, hence $\tilde{F}_{n}$ contracts in $\tilde{X}$ (since $\bar{F}_{n}$ contracts in $X$ ), hence $\tilde{X}$ is 1-connected (exactness), hence $\tilde{F}_{n} \rightarrow \bar{F}_{n}$ is a weak homotopy equivalence (exactness), and $\operatorname{dim}_{Z} \bar{F}_{n}<\infty$ by Lemma 2.2 .

Similarly, but more easily, $\operatorname{dim}_{Z} \tilde{G}_{n}<\infty$. Clearly $\operatorname{dim}_{Z} \tilde{X}_{n}<\infty$. $X$ is homotopy equivalent to a finite-dimensional complex (a simple argument using [11] is given above). Hence Lemma 2.4 gives $\operatorname{dim}_{Z} \tilde{X}<\infty$.

Lemma 2.3 then gives $\operatorname{dim}_{Z} \tilde{X}_{n} \leqslant \operatorname{dim}_{Z} \tilde{X}_{n+1} \leqslant \operatorname{dim}_{Z} \tilde{X}<\infty$. Thus, for large $n, \operatorname{dim}_{Z} \tilde{X}_{n}=\operatorname{dim}_{Z} \tilde{X}_{n+1}$ so that $\operatorname{dim}_{Z} \tilde{G}_{n}=0$, (again by Lemma 2.3). But $\tilde{G}_{n}$ is path-connected and $\pi_{1}\left(\tilde{G}_{n}\right)$ is abelian. So $\tilde{f}_{n}$ is a weak homotopy equivalence for large $n$. Now $\pi_{i}(\tilde{X}) \rightarrow$ proj $\lim _{n} \pi_{i}\left(\tilde{X}_{n}\right)$ is clearly onto. So $\tilde{p}_{n \#}$ : $\pi_{i}(\tilde{X}) \rightarrow \pi_{i}\left(\tilde{X}_{n}\right)$ is onto for large $n$. But, as we saw, $\tilde{F}_{n}$ contracts in $\tilde{X}$. So $\tilde{p}_{n \#}$ is also $1: 1$. So $\tilde{p}_{n}$ is a weak homotopy equivalence. Hence $\bar{p}_{n}$ is too. Hence $\bar{p}_{n}$ is an unpointed homotopy equivalence. 
3. Proof of Theorem 1.2. We are given, for all $n$, homotopy commutative diagrams

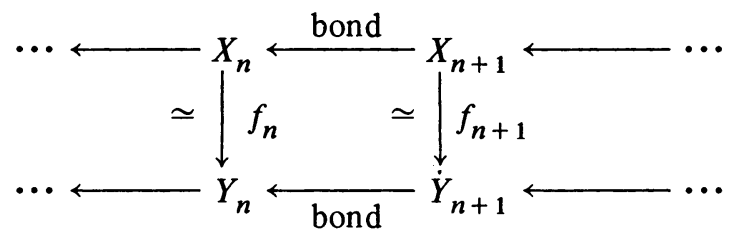

with $f_{n}$ a fibration.

Form the following diagram (compare [6, p. 178]):

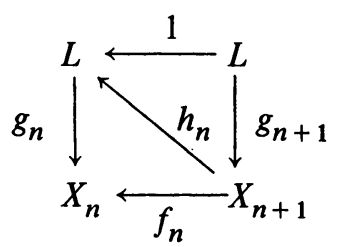

Here, the third horizontal sequence is formed from the corresponding "unbarred" sequence by inductively replacing the maps $h_{n}$ and $g_{n+1}$ by fibrations $\bar{h}_{n}$ and $\bar{g}_{n+1}$. See, for example, [4, p. 526] for this standard operation. The second and fourth lines are cofinal subsequences of the third. The vertical arrows, marked $\simeq$, are homotopy equivalences which are chosen (using the fibration property of $f_{n}$ and $1_{L}$ ) to make the whole diagram strictly commute.

The second, third and fourth lines have inverse limits homeomorphic to some space $Z$. It follows from Theorem 3.1, below, that the vertical arrows, marked $\simeq$, induce homotopy equivalences $Z \rightarrow L$ and $Z \rightarrow X$; hence $X$ is homotopy equivalent to $L$.

THEOREM 3.1. Suppose given a strictly commutative diagram of spaces

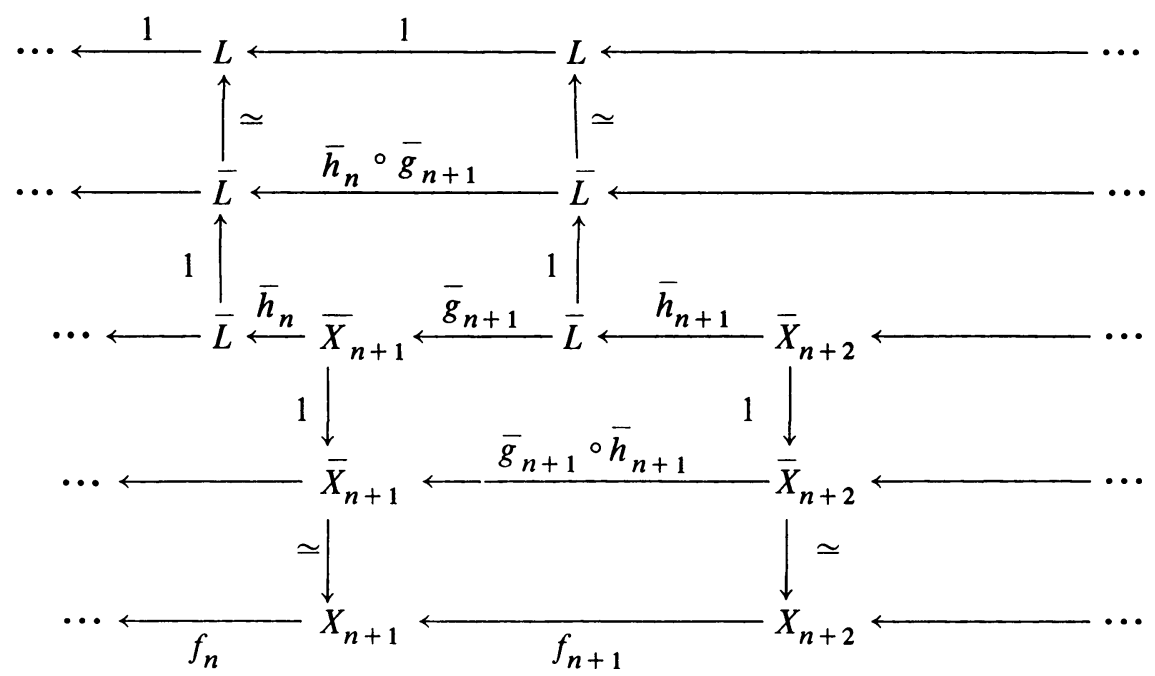


in which each $f_{n}$ is a homotopy equivalence and each bond is a fibration, then the inverse limit map $f: X \rightarrow Y$ is a homotopy equivalence.

This is a restatement of Theorem 3.4.1 of [6]. The reader can write down for himself a three-page elementary proof of Theorem 3.1 by carefully reading Chapter 3 of [6], Chapter 1 of [14] and Theorem 3.1 of [9]. Or see [21].

4. Proof of Theorem 1.3. We are now given that each $X_{n}$ is a compact metric ANR. By Theorem 1.2, $X$ is homotopy equivalent to a complex, so we can use the proof of Theorem 1.1. In the notation of $\$ 2, \bar{f}_{n}$ is both a fibration and a weak homotopy equivalence for large $n$. Hence (Lemma 2.2) each $\bar{f}_{n}$ has contractible point-inverses. Let $\bar{X}=\operatorname{proj} \lim _{n}\left\{\bar{X}_{n} ; \bar{f}_{n}\right\}$, and let $Q$ be the Hilbert Cube. $\bar{X}_{n}$ is a compact ANR, so $\bar{f}_{n} \times 1: \bar{X}_{n+1} \times Q \rightarrow \bar{X}_{n} \times Q$ is the uniform limit of homeomorphisms between $Q$-manifolds [2, Theorems 44.1 and 43.1]. Hence [1], $\bar{X} \times Q$ is homeomorphic to $\bar{X}_{n} \times Q$ for large $n$, and therefore $\bar{X}$ is a compact ANR. Since $X$ is path connected, the fibration with unique path lifting $\bar{X} \rightarrow X$ is a covering projection [16, p. 81]. So $X$ is a compact ANR.

\section{REFERENCES}

1. M. Brown, Some applications of an approximation theorem for inverse limits, Proc. Amer. Math. Soc. 11 (1960), 478-483.

2. T. A. Chapman, Lectures on Hilbert cube manifolds, CBMS Regional Conf. Ser. in Math., vol. 28, Amer. Math. Soc., Providence, R.I., 1976.

3. T. tom Dieck, Partitions of unity in homotopy theory, Compositio Math. 23 (1971), 159-167.

4. D. A. Edwards and R. Geoghegan, Shapes of complexes, ends of manifolds, homotopy limits and the Wall obstruction, Ann. of Math. (2) 101 (1975), 521-535; correction 104 (1976), p. 389.

5. Amer. Math. Soc. 214 (1975), 261-277.

6. D. A. Edwards and H. M. Hastings, $\check{C}$ ech and Steenrod homotopy theories with applications to geometric topology, Lecture Notes in Math., vol. 542, Springer-Verlag, Berlin and New York, 1976.

7. R. Geoghegan, Compacta with the homotopy type of finite complexes, (Proc. Georgia Conf. on Geometric Topology, 1977), Academic Press, New York (to appear).

8. R. Geoghegan and R. C. Lacher, Compacta with the shape of finite complexes, Fund. Math. 92 (1976), 25-27.

9. H. M. Hastings, Fibrations of compactly generated spaces, Michigan Math. J. 21 (1974), 243-251.

10. S. Mardešić and J. Segal, Shapes of compacta and ANR-systems, Fund. Math. 72 (1971), 41-59.

11. M. Mather, Counting homotopy types of manifolds, Topology 4 (1965), 93-94.

12. J. Milnor, On spaces having the homotopy type of $C W$ complexes, Trans. Amer. Math. Soc. 90 (1959), 272-280.

13. K. Morita, On shapes of topological spaces, Fund. Math. 86 (1975), 251-259.

14. D. G. Quillen, Homotopical algebra, Lecture Notes in Math., vol. 43, Springer-Verlag, Berlin and New York, 1967.

15. L. C. Siebenmann, L. Guillou and H. Hähl, Les voisinages réguliers ouverts, Ann. Sci. Ecole Norm. Sup. (4) 6 (1973), 253-293.

16. E. H. Spanier, Algebraic topology, McGraw-Hill, New York, 1966.

17. A. Strom, Note on cofibrations, Math. Scand. 19 (1966), 11-14.

18. C. T. C. Wall, Finiteness conditions for CW-complexes, Ann. of Math. (2) 81 (1965), 55-69. 
19. J. E. West, Mapping Hilbert cube manifolds to ANRs, Ann. of Math. (2) 106 (1977) (to appear).

20. J. H. C. Whitehead, A certain exact sequence, Ann. of Math. (2) 52 (1950), 51-110.

21. R. Geoghegan, The inverse limit of homotopy equivalences between towers of fibrations is a homotopy equivalence-a simple proof (submitted).

Department of Mathematics, State University of New York, Center at Binghamton, Binghamton, NeW YoRK 13901 